\title{
BMJ Open Study protocol for a feasibility study of an internet-administered, guided, CBT- based, self-help intervention (ENGAGE) for parents of children previously treated for cancer
}

To cite: Woodford J, Wikman A, Cernvall M, et al. Study protocol for a feasibility study of an internet-administered, guided, CBT-based, self-help intervention (ENGAGE) for parents of children previously treated for cancer. BMJ Open 2018;8:e023708. doi:10.1136/ bmjopen-2018-023708

- Prepublication history and additional material for this paper are available online. To view these files, please visit the journal online (http://dx.doi org/10.1136/bmjopen-2018023708).

Received 19 April 2018

Revised 3 May 2018

Accepted 4 May 2018

\section{Check for updates}

${ }^{1}$ Clinical Psychology in Healthcare, Department of Women's and Children's Health, Uppsala University, Uppsala, Sweden

${ }^{2}$ Paediatric Oncology, Department of Women's and Children's Health, Uppsala University, Uppsala, Sweden

Correspondence to Dr Joanne Woodford; joanne.woodford@kbh.uu.se

\section{ABSTRACT}

Introduction A subgroup of parents of children previously treated for cancer report long-term psychological distress after end of treatment. However, needs for psychological support are commonly unmet and there is a lack of evidence-based treatments tailored to the specific needs of this population. An internet-administered, guided, cognitive-behavioural therapy-based, self-help intervention (ENGAGE) for parents of children previously treated for cancer may provide a solution. The aim is to examine the feasibility and acceptability of the intervention ENGAGE and the study procedures for a future controlled trial.

Methods and analysis The study has an uncontrolled within-group design with an embedded qualitative and quantitative process evaluation. Potential participants are parents of children previously treated for cancer, living in Sweden, recruited via their child's personal identification number (via the Swedish Childhood Cancer Registry and the Swedish Tax Agency). Parents are invited randomly with information packs sent to home addresses. Further interest in participating can be registered via information on relevant websites. The study aims to recruit 50 parents who will receive the intervention ENGAGE which is designed to be delivered over a 10week period, and comprises one introductory chapter followed by up to 10 intervention modules addressing key concerns identified for the population. Consistent with feasibility study objectives, primary outcomes relate to recruitment, attrition, data collection, study resources, intervention delivery and acceptability. Clinical outcomes (post-traumatic stress, depression, anxiety, fear of cancer recurrence, psychological inflexibility and experiential avoidance, depressed inactivity, fatigue, quality of life and self-compassion) will be measured at baseline, posttreatment (12 weeks) and 6-month follow-up.

Ethics and dissemination The Regional Ethical Review Board in Uppsala, Sweden has granted approval for the study (Dnr: 2017/527). Results will be disseminated to relevant healthcare and patient communities, in peerreviewed and popular science journals, and at scientific and clinical conferences.

Trial registration number ISRCTN57233429; Pre-results.

\section{Strengths and limitations of this study}

Development of the internet-administered, guided cognitive-behavioural therapy-based, self-help intervention ENGAGE has included consulting of theoretical literature and clinical expertise and extensive involvement of end-users by means of participatory action research.

- This study will examine the feasibility and acceptability of the intervention ENGAGE alongside planned study procedures for a controlled trial.

- This study will examine methodological, procedural and clinical challenges to revise and refine the intervention ENGAGE, study design and planned procedures prior to a controlled trial following established feasibility study progression criteria.

- This study is limited to examining the feasibility and acceptability of intervention delivery within a university setting and does not examine the feasibility of implementation within a real-life healthcare context.

\section{INTRODUCTION}

Most children diagnosed with cancer survive their disease. ${ }^{1}$ However, childhood cancer impacts on the whole family from diagnosis into survivorship. ${ }^{2}$ For parents, a child's treatment completion is an important milestone and a period of psychological vulnerability. ${ }^{34} \mathrm{~A}$ subgroup reports negative long-term psychological consequences years after treatment completion. $^{4-7}$ However, there is a lack of evidence-based psychological interventions for parents who experience distress in relation to a child's cancer disease after end of treatment. Recently published guidelines, informing how children diagnosed with cancer and their family members should be cared for, recommend referrals to appropriate therapeutic support into long-term survivorship. ${ }^{8}$ However, significant challenges 
remain regarding provision of such support. We have reported an unmet need of psychological support among parents of children previously treated for cancer. ${ }^{9}$ The results are in line with findings from an Australian study showing that formal psychological support was difficult to access and rarely received by parents after completion of cancer treatment. ${ }^{10}$ Factors related to staff availability, models of assessment and delivery of services, and size and location of paediatric cancer units hindered provision of support.

Using the internet to deliver psychological support may increase access to support and represent an alternative model of psychological support delivery for parents of children previously treated for cancer. We have shown an internet-administered psychological, self-help intervention to be effective in reducing symptoms of anxiety, depression and post-traumatic stress (PTSS) among parents of children recently diagnosed with cancer, ${ }^{11}$ with improvements maintained at 1-year follow-up. ${ }^{12}$ However, challenges with recruitment and attrition were encountered, indicating that end-user involvement in the development of interventions, alongside informing study procedures to test and evaluate interventions, may be essential for intervention research within this population. ${ }^{13}$ Research suggests internet-administered, self-help interventions should be developed with the target population in mind, ${ }^{14}$ with lower levels of acceptability found for internet-administered interventions not developed for a specific population. ${ }^{15}$ Additionally, recruitment and adherence rates may improve if the perspective of the population is adopted. ${ }^{16}$ Finally, there is currently a lack of evidence-based psychological support for parents who experience distress in relation to a child's cancer disease after successful cancer treatment, despite clear findings showing that a subgroup of parents report long-term psychological distress. ${ }^{45}$

The aim is to examine the feasibility and acceptability of the internet-administered, cognitive-behavioural therapy (CBT)-based, guided, self-help intervention ENGAGE for parents of children previously treated for cancer and the study procedures for a future controlled trial. We have undertaken a programme of phase I (development) research, following the Medical Research Council complex interventions framework ${ }^{17}$ to inform the development of the intervention. First, a systematic review of cancer-related long-term negative and positive psychological effects for parents of childhood cancer survivors was conducted, ${ }^{4}$ with results used to inform the development and piloting of a face-to-face CBT-based intervention. The intervention was successful, resulting in improvements in symptoms of anxiety, depression and PTSS $(\mathrm{d}=0.65-0.92)$ at post-treatment and 3-month follow-up. ${ }^{18}$ Subsequently, a participatory action research (PAR) ${ }^{19}$ approach was adopted to inform the design, content and delivery of the intervention in collaboration with parent and expert research partners. ${ }^{20}$ Finally, an online survey study, utilising a population-based cross-sectional design, further examined preferences regarding study procedures, for example, type of controlled design and mode of recruitment (letter vs postal card) ('Personal communication' by J Woodford, 20180406). Parents of children who had completed cancer treatment were invited to complete the survey, with $32 \% \quad(n=112)$ of 350 responding. Findings indicated no differences in response rate between mode of invitation $(n=59(34 \%)$ letter, $\mathrm{n}=53(30 \%)$ postal card; $\mathrm{p}=0.447)$. Overall, parents perceived proposed study procedures (eg, receipt of initial study information via postal letter, presentation of detailed study information online, via text and informational video, randomisation) and the receipt of internet-administered psychological support as acceptable. These findings informed the recruitment and provision of study information procedures to be further examined in the present feasibility study.

The key feasibility outcomes examined via the proposed protocol concern methodological, procedural and clinical uncertainties, ${ }^{21-23}$ including: (1) estimates of likely recruitment and retention rates; (2) feasibility and acceptability of data collection instruments and data collection procedures; and (3) feasibility and acceptability of the intervention. In line with standard feasibility study objectives, ${ }^{21}$ improvements regarding clinical outcomes are not examined at this stage. However, an embedded qualitative process evaluation ${ }^{24}$ will be used to examine the: (1) acceptability of intervention; (2) self-reported psychological needs; (3) parents' healthcare utilisation and productivity losses related to the child's cancer disease; (4) potential mechanisms of change; and (5) the impact of the intervention on parents' difficulties.

\section{METHODS AND ANALYSIS}

This protocol (version 1, 14 March 2018) is reported according to guidelines presented in the Consolidated Standards of Reporting Trials (CONSORT) 2010 statement extension for randomised pilot and feasibility studies $^{23}$ and clinical trial protocols. ${ }^{25}$

\section{Design}

The study has an uncontrolled, within-group, baseline, post-treatment (12 weeks) and 6-month follow-up design with an embedded qualitative and quantitative process evaluation. All participants will receive the internet-administered, CBT-based, self-help intervention ENGAGE, guided by an e-therapist for 10 weeks, comprising one introductory chapter followed by up to 10 treatment modules addressing key concerns identified for the population.

\section{Eligibility criteria}

Parents/caregivers (from here referred to as parents or participants) will be included according to: (1) parent of a child diagnosed with cancer when $0-18$ years who has completed cancer treatment 3 months to 5 years previously; (2) resides in Sweden; (3) able to read and understand text in Swedish; (4) has access to email, the internet 
and a mobile telephone and/or Bank ID (a citizen authentication system used in Sweden); and (5) self-reports a need for psychological support related to the child's cancer disease and treatment. The following exclusion criteria will be used: (1) self-reported or clinician assessed (based on the Mini-International Neuropsychiatric Interview, MINI) ${ }^{26}$; symptoms of a severe and enduring mental health difficulty; (2) self-reported or clinician assessed (based on the MINI neuropsychiatric interview) ${ }^{26}$; misuse of alcohol, street drugs and/or prescription medication; (3) acutely suicidal; and (4) currently attending psychological treatment. Those excluded due to a severe and enduring mental health difficulty, substance misuse and/ or acute suicidality will be guided to appropriate healthcare services.

\section{Sample size}

The eligible population includes approximately 2400 parents, with around 30\% (720/2400) expected to experience a need of psychological support..$^{9}$ Approximately $30 \%$ of these $(216 / 720)$ are expected to potentially consent. ${ }^{11} 1218$ Following recommendations of sample sizes of 50-60 being appropriate to assess feasibility outcomes and estimate sample size for a definite trial ${ }^{27}$ we aim to recruit a sample of 50 participants. If 50 are included, we will be able to estimate a participation rate of $90 \%$ within a $95 \%$ CI of $\pm 8 \%$.

\section{Recruitment}

Participants will be recruited using two approaches: (A) Children's personal identification numbers will be obtained from the Swedish Childhood Cancer Registry (National Quality Registry, initiated in 1982) and linked to parents' names and addresses via NAVET, a registry held by the (Swedish Tax Agency). Parents will be invited to participate randomly by the research team, using blocks of 100 until the target number of 50 has been reached. Prior to inviting parents into the study, the most up-to-date information concerning whether children are currently living or deceased will be checked via the telephone by a member of the research team with Swedish Tax Agency. A study information pack will be sent to home addresses, including brief information about the study and a www address to a secure website, the U-CARE portal (Portal). Potential participants will be able to access information via the Portal, with study information presented in text and video format. They will be able to either register interest in the study, or opt out of the study, by: (1) completing an online form via the Portal; (2) returning a reply slip using a freepost envelope; (3) telephoning the research team; or (4) emailing the research team. Given the use of reminders improves recruitment rates, ${ }^{28}$ telephone numbers of those who do not respond to the research team, within 4 weeks of sending the postal study information pack, will be identified via internet search engines. A member of the research team will telephone all non-responders. The purpose will be to confirm receipt of the study invitation pack and answer any questions the parent may have regarding the study. In cases where the telephone call is not answered a maximum of four additional telephone call attempts to establish contact will be made over the following 4 weeks. The study information sheet will clearly specify that a member of the research team will attempt to telephone call non-responders, with parents provided with the aforementioned methods of opting out of the study should they not wish to receive a telephone call from a member of the research team. In cases where a participant's telephone number cannot be identified, a study information pack will be resent via the post with a reminder note added to the pack by the research team if no response is received within 4 weeks. (B) To raise awareness of the study, and potentially recruit to the study, advertisements will be placed on relevant social media sites and patient organisations' and interest groups' websites. People can receive more information about the study by telephoning or emailing the research team and register interest in the study by completing an online form via the Portal.

\section{Reasons for non-participation}

Parents deciding to opt out of further contact will be presented a short questionnaire (provided in paper format in information packs as well as online) listing possible reasons for non-participation as informed by previous research ${ }^{2930}$ alongside an open-ended question for parents to provide further information and reasons for non-participation should they wish. Reasons for non-participation will be used to inform about barriers to recruitment and may provide data pertaining to the acceptability of the intervention and support offered. It will be made clear on both the paper and online questionnaire that the provision of reasons for non-participation is optional and parents do not need to report why they do not wish to participate if they would prefer not to.

\section{Informed consent, screening and baseline}

Parents interested in participating will be asked to provide informed consent and contact details via the Portal. Those who reply to the research team via a postal reply slip, telephone or email will be called by a member of the research team, who will provide more information about the study. Those interested in participating will be asked to provide consent via the Portal. Parents who speak to a member of the research team and express interest in the study, but do not provide online consent within 2 weeks, will receive a telephone follow-up call and/or email from a member of the research team. Where telephone calls are not answered, a maximum of five telephone calls will be made over a period of 2 weeks.

Parents providing informed consent will be contacted via telephone by a licensed psychologist for an eligibility interview with the purpose of confirming inclusion and exclusion criteria. If eligibility is confirmed, parents will 
be instructed to complete a baseline assessment via the Portal, with the option provided to complete the assessment via the telephone if preferred. In addition, a semistructured interview will be completed at baseline over the telephone to gain a more detailed understanding, for example, concerning presenting problems and expectations for treatment. After the full baseline assessment is completed, parents will be provided access to the ENGAGE intervention via the Portal and will be allocated to an e-therapist. Participant flow through the study is illustrated in figure 1.

\section{Intervention}

Content

Based on standard definitions of self-help ${ }^{31}$ and taxonomies categorising levels of support, ${ }^{32}$ ENGAGE can be described as an internet-administered, guided, CBT-based, self-help intervention. ENGAGE includes written, audio and video materials provided online via the Portal. The intervention includes: (1) a short introductory chapter, followed by 10 CBT-based modules, with a brief overview of module content shown in table 1; (2) an initial assessment session with an e-therapist via a video or telephone call during which the individual's problems and idiographic goals are formulated and parents are directed to the short introductory chapter and first module; and (3) weekly guidance from an e-therapist via the Portal (online written feedback). The intervention is designed to be delivered over a 10-week period, with parents encouraged to complete one module per week. However, the intervention will be accessible to parents for a 12-week period to provide flexibility regarding module completion and provision of e-therapist feedback and support. Module content is based on CBT techniques and is tailored towards key concerns and difficulties experienced by parents of children previously treated for cancer as informed by previous research. ${ }^{3-5} 18$ Each module includes psychoeducation alongside text, audio and video material instructing parents in the use of specific CBT-based techniques. Parents will be encouraged to complete weekly action plans and symptom questionnaires for each module which will be reviewed by the e-therapist. Further, modules include case vignettes, serving to clarify important treatment principles and help parents make connections between the material and their own experiences. Extensive efforts have been made to include 'common factors' within the intervention in order to establish, develop and maintain a therapeutic alliance. ${ }^{33}$ As such, the intervention has been developed to engage parents in materials by including statements of empathy, genuineness and warmth, narratives referring to struggle and recovery, examples to help parents relate the text material to their own lives and personal metaphors for emotional distress. ${ }^{33}$

\section{Guidance from e-therapists}

An e-therapist will guide the use of the intervention, following a structured support protocol developed specifically for the intervention. Guidance will consist of one video or telephone assessment session, weekly online written support and a mid-treatment video or telephone 'booster' session. Prior to the start of the intervention parents will be contacted by an e-therapist for the individual assessment session via video call, lasting approximately $45 \mathrm{~min}$. For parents not wanting to participate in a video call there is also an option to complete the call via telephone. Parents will be able to communicate their current difficulties and the e-therapist will conduct an individually tailored behaviour analysis of the parent's main difficulties, collaboratively formulate idiographic treatment goals and introduce the intervention. Although parents will work independently with the intervention, e-therapists will provide weekly, and at-need, written support messages online via the Portal throughout the intervention. E-therapist guidance will include the provision of feedback on action plans, reinforcement of progress made, validating any difficulties experienced and providing assistance problem-solving difficulties. E-therapists will provide encouragement, motivation and guidance throughout the intervention, with parents able to contact their allocated e-therapist for additional guidance, via the Portal or telephone, should they experience a need. E-therapists will be obliged to respond to parents within one working day. Given the explorative nature of the study, no maximum time limit for support has been set, though based on previous experience ${ }^{1112}$ we anticipate e-therapists will spend approximately 20-30 min per parent each week.

Parents will be provided a 'booster' session lasting 30-45 min, via video or telephone call, mid-way through the intervention. This session will be an opportunity to further assess any potential difficulties experienced with the ongoing work, provide additional guidance and assistance problem-solving, alongside the provision of encouragement and motivation.

Parents who do not log in, or show low activity in the intervention, will be contacted via text message, email and/or telephone, whichever is preferred by the parent and e-therapist.

\section{E-therapists}

E-therapists will be psychology programme students, in at least their fourth year of study, having completed a minimum of their first term of advanced studies in CBT, but will have not yet begun their prescribed practical service (ie, praktisk tjänstgöring för psykologer). Prior to study start, all e-therapists will participate in a 1-day workshop to familiarise themselves with the treatment manual and support protocol, delivered by a licensed clinical psychologist. E-therapists will receive weekly group clinical supervision sessions focusing on case discussions, skills development and at-need supervision by a licensed clinical psychologist with relevant experience of the population. 


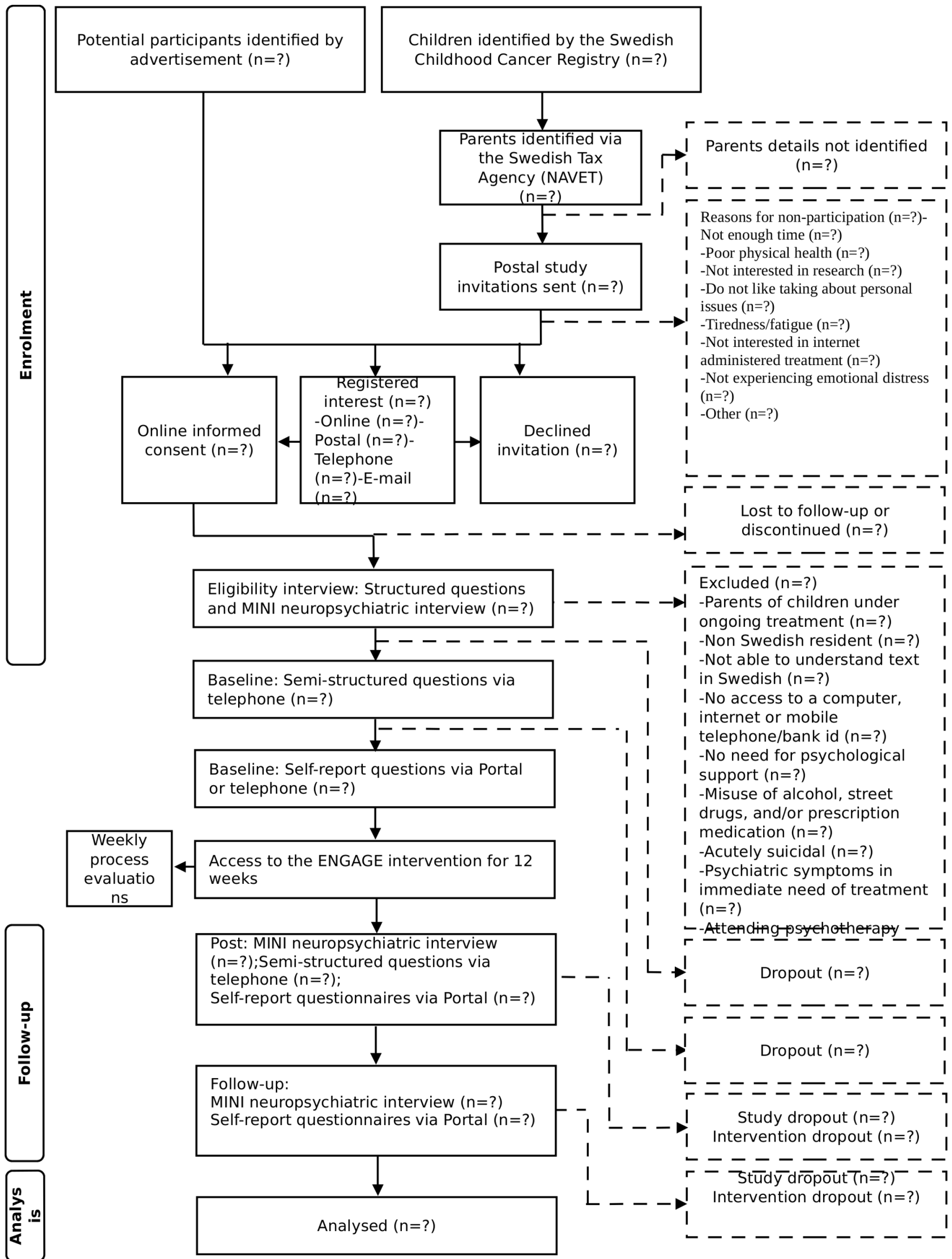

Figure 1 Consolidated Standards of Reporting Trials (CONSORT) diagram. MINI, Mini-International Neuropsychiatric Interview. 
Table 1 Overview of the 10 modules included in the ENGAGE intervention

\section{Title and description}

Module $1 \quad$ 'What have I experienced and where am I
heading?'
Processing and normalising the cancer experience
and cancer-related distress, goal setting.

Module 2

'Who takes care of me?'

Analysing current problems using functional analysis. Principles of self-compassion.

$\begin{array}{ll}\text { Module } 3 & \begin{array}{l}\text { 'Am I really here?' } \\ \text { Mindfulness and acceptance-based strategies. }\end{array} \\ \text { Module } 4 & \text { 'Painful experiences' } \\ & \begin{array}{l}\text { Exposure to painful memories and emotions and } \\ \text { introducing skills to handle challenging situations } \\ \text { and experiences. }\end{array}\end{array}$

Module $5 \quad$ 'Looking inwards'
Managing emotional avoidance through exposure.

Module 6

'The worst I've ever experienced'

Deepened focus on painful memories and emotions through expressive writing.

\section{Cognitive behavioural therapy strategies}

Psychoeducation about typical reactions among parents of children previously treated for cancer. Setting intervention goals and long-term goals. Identifying challenging situations.

Introducing functional analysis. Psychoeducation about self-compassion and difficult life events. Practising self-compassion and functional analysis.

Psychoeducation about emotions and mindfulness. Practising noticing emotions and bodily sensations. Mindfulness and acceptance-based exercises and continue practising functional analyses.

Psychoeducation about painful memories and emotions, and coping with fear of recurrence. Rationale for exposure techniques. Cognitive strategies for disengaging from patterns of unhelpful thinking. Continue practising mindfulness and functional analyses.

Intensifying exposure with specific focus on emotional avoidance through functional analyses and further exposure techniques. Continue practising mindfulness.

Continued psychoeducation about painful memories and emotions. Reflecting on exposure exercises, reviewing goals from start of the programme. Continue practising mindfulness. Expressive writing task.

Reviewing goals and identifying challenging situations that remain, plan of action. Rationale for behavioural activation. Continuing exposure exercises.

Continued psychoeducation about behaviour activation and self-compassion. Continue to practising self-compassion and behavioural activation.

Module 9
$\begin{aligned} & \text { 'Becoming your own therapist' } \\ & \text { Applying new skills flexibly in everyday life. }\end{aligned}$
Module 10
$\begin{aligned} & \text { 'Where have I been and where am I heading now?' } \\ & \text { Progress review and skills for maintaining progress } \\ & \text { and setbs. }\end{aligned}$

Psychoeducation about becoming one's own therapist. Identifying challenging situations that remain, review goals and form action plans. Focus on applying new skills in everyday life in a flexible manner.

Reviewing the intervention, what worked better/ worse, skills for maintaining change and handling setbacks.

\section{Optional support functions}

Optional support functions within the Portal will include an online library containing information about CBT, selfhelp, literature suggestions, links to relevant websites, as well as CBT exercises from the intervention. These materials will be available as downloadable text and audio files. In addition, all exercises used in the intervention will be accessible within the library for parents to revisit. As these functions are optional and not part of the treatment, parents will not receive any recommendations from their e-therapist regarding optimal level of engagement with these support functions.

\section{Setting}

Parents will receive guidance from e-therapists located at Uppsala University, Sweden. Due to the nature of the ENGAGE intervention being online, parents are anticipated to engage with the intervention in their own homes.

\section{Outcome measures}

Feasibility outcomes

Feasibility outcomes of interest relate to methodological, procedural and clinical uncertainties ${ }^{21-23}$ and examine recruitment rates, eligibility criteria, data collection, attrition, resources needed to complete the study and 
Table 2 Overview of feasibility outcomes and progression criteria

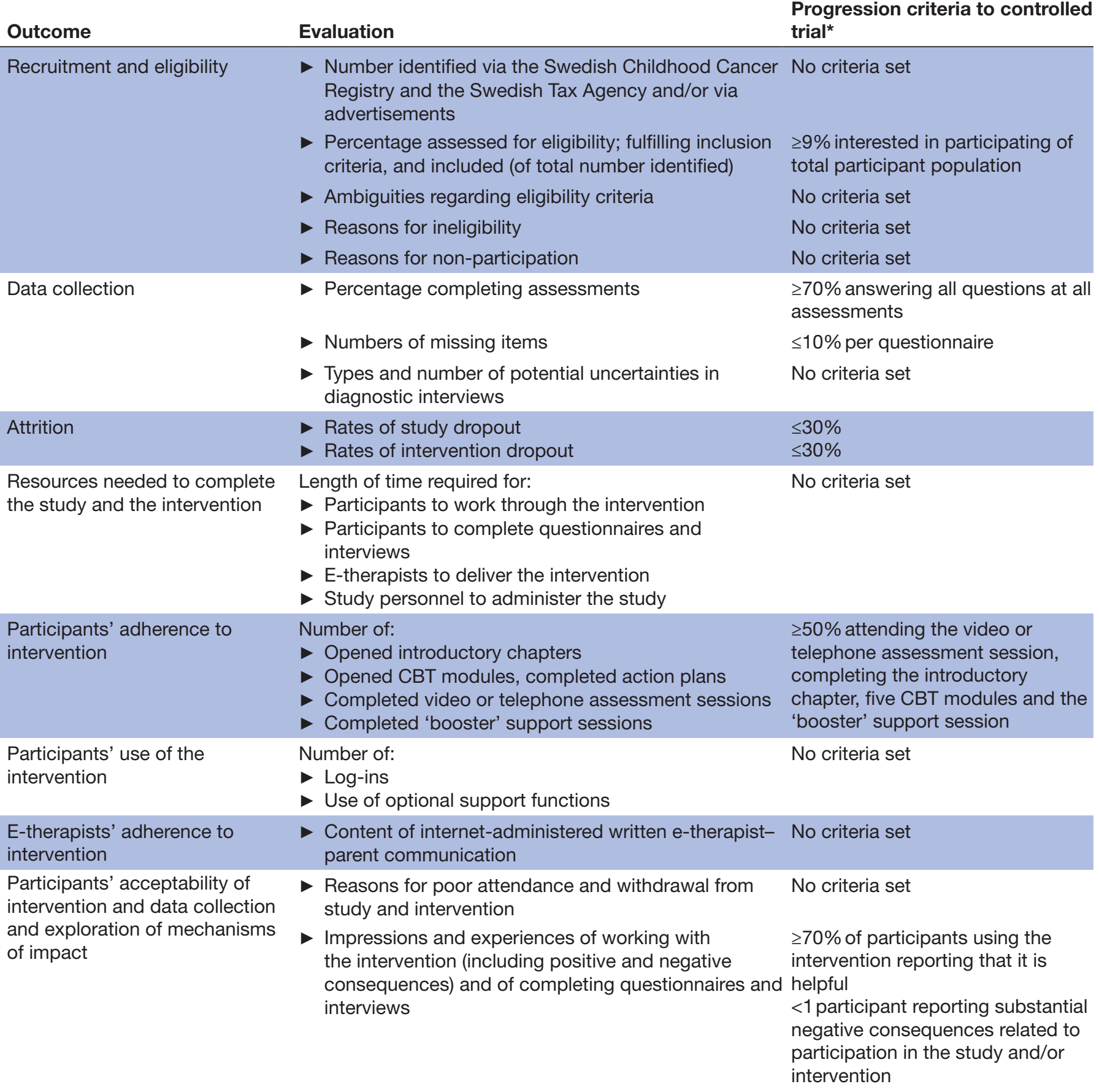

*If one or more criteria are not met revisions should be considered before proceeding to a controlled trial.

CBT, cognitive-behavioural therapy.

intervention, parents' adherence to the intervention, e-therapists' adherence to the support protocol and parents' acceptability of the intervention and study procedures. Feasibility outcomes assessed are shown in table 2 alongside the associated progression criteria (where applicable). Progression criteria have been set to facilitate the interpretation of results and to inform whether to proceed to a definite trial after the feasibility study.

\section{Sociodemographic and clinical variables}

Data on child age, gender, diagnosis, date of first diagnosis, date of end of treatment (where available) and type of treatment will be obtained from the Swedish Childhood Cancer Registry. Self-report data on parent age, gender, education, employment status, ethnicity, relationship status, number of children, ages of children, current housing situation, previous psychological treatment, previous traumatic events, physical health, date of 
end of child's cancer treatment, cancer recurrence and parents' experience using the internet will be collected at the eligibility interview.

\section{Psychological and health economics outcomes}

PTSS will be assessed using the revised Post-traumatic Stress Disorder Checklist for DSM-5 (PCL-5) ${ }^{34}$ and the DSM-IV (PCL-C) ${ }^{35}$ for comparison with our previous studies. ${ }^{5} 111218$ Symptoms of depression will be assessed using the Patient Health Questionnaire (PHQ-9), ${ }^{36}$ the Generalized Anxiety Disorder 7-item scale ${ }^{37}$ will be used to assess symptoms of anxiety. Frequency of parental fear of cancer recurrence and of their child experiencing another serious health condition will be measured by five-item Likert scales developed by the study authors LvE, JW and AW, and rated from 'very often' to 'not at all'. Psychological inflexibility and experiential avoidance will be measured with the Acceptance and Action Questionnaire, 6-items (AAQ-6) ${ }^{38}$ The Behavioural Activation for Depression Scale (BADS) ${ }^{39}$ will be used to measure depressive inactivity. Symptoms of fatigue will be measured with the Fatigue Severity Scale, ${ }^{40}$ and the Self-Compassion Scale-Short Form ${ }^{41}$ will be used to measure self-compassion. The EuroQol 5-dimension questionnaire ${ }^{42}$ will be used to assess quality of life, with impact on use of healthcare services, employment, absence and sick leave examined with a modified short version of the Trimbos and Institute of Medical Technology Assessment Cost Questionnaire for Psychiatry, ${ }^{43}$ assessing direct and indirect medical costs and indirect non-medical costs. The MINI neuropsychiatric interview ${ }^{26}$ will be used to assess current psychiatric disorders.

\section{Data collection}

Data will be collected via telephone and/or the Portal at the eligibility interview, baseline, post-treatment (12 weeks) and at 6-month follow-up. In order to minimise attrition, prompts will be sent to indicate it is time to complete the next assessment, with parents able to indicate how they would prefer to receive prompts (eg, via email, telephone, text message or post). Additional reminders will be sent when assessments have not been completed within 1 week following a prompt. If parents do not complete post-treatment (12 weeks) and 6-month follow-ups on the Portal within 2 weeks of receiving the first prompt, they will be provided with an option to complete the outcome measurements over the telephone with a member of the research team. A study newsletter will be sent to participants via email approximately 6 weeks before post-treatment (12 weeks) and 6-month follow-up, given evidence suggesting using study newsletters as a prenotification device can improve follow-up rates. ${ }^{44}$ Study newsletter content will change during the course of the study and will include both current study-specific and wider research group news, alongside a reminder that the next assessment will be due in 6 weeks.

Weekly measures of PTSS (PCL-5; PCL-C), depression (PHQ-9), experiential avoidance (AAQ-6) and depressed inactivity (BADS) will be collected via the Portal during the intervention to examine the feasibility of collecting quantitative process evaluations data. All measurements collected during the course of the study, including mode of administration at each assessment, are shown in table 3.

\section{Participant adherence}

Opened modules, completed action plans and private messages to e-therapists will be logged via the Portal to examine adherence to the intervention. Further, total number of log-ins and use of optional support functions will be logged to examine use of the intervention. Full adherence to the intervention will be defined as: (1) attendance of the initial individual assessment session, via video or telephone; (2) completion of the introductory chapter; (3) completion of five CBT modules, as defined by submission of each associated action plan to the e-therapist; and (4) attendance of the 'booster' support session.

\section{E-therapist adherence}

To assess e-therapist adherence, improve training and identify areas requiring further modification or development, all video and telephone support sessions will be recorded with parent consent and reviewed by a clinical supervisor external to the research team. In addition, email communication will be reviewed by the clinical supervisor to further ensure adherence to the intervention and adequacy of e-therapist competency. Furthermore, a $15 \%$ sample of the written and $15 \%$ of video/ telephone communication between e-therapists and parents will be reviewed for e-therapist adherence and competence in supporting the intervention according to an adherence measure developed for the ENGAGE intervention, performed by a member of the research team (with relevant clinical expertise), otherwise not associated with the study.

\section{Qualitative process evaluation}

Semistructured interviews will be conducted by a psychologist with parents via the telephone at baseline and post-treatment (12 weeks). Sample size cannot be stated a priori and interviews will be conducted until data saturation is met.

Baseline: Participants will be asked to describe their main presenting psychological difficulties and related needs, and expectations concerning the ENGAGE intervention. To inform any future health economic evaluation, they will be asked to describe distressing concerns regarding healthcare utilisation and productivity losses related to their child's cancer disease.

Post-treatment: Parents will be interviewed to explore the acceptability of the ENGAGE intervention and associated study procedures. In order to examine possible mechanisms of change, parents' views concerning the impact of the ENGAGE intervention and CBT techniques had on their mood, and lives more generally, will be explored. The interview guide will be informed by previous research examining the acceptability of CBT self-help 
Table 3 Overview of measures at the respective assessments

\begin{tabular}{|c|c|c|c|c|c|c|c|}
\hline Variable/phenomena & Measure & $\begin{array}{l}\text { Eligibility } \\
\text { interview }\end{array}$ & Baseline & Postassessment & $\begin{array}{l}\text { Weekly } \\
\text { process } \\
\text { evaluation }\end{array}$ & $\begin{array}{l}\text { Six-month } \\
\text { follow-up }\end{array}$ & $\begin{array}{l}\text { Mode of } \\
\text { administration }\end{array}$ \\
\hline $\begin{array}{l}\text { Child age, gender, } \\
\text { diagnosis, date of first } \\
\text { diagnosis, date of end } \\
\text { of treatment (where } \\
\text { available) and type of } \\
\text { treatment }\end{array}$ & $\begin{array}{l}\text { The Swedish } \\
\text { Childhood } \\
\text { Cancer Registry }\end{array}$ & & & & & & $\begin{array}{l}\text { Swedish Childhood } \\
\text { Cancer Registry } \\
\text { (recruitment strategy } \\
\text { A)/telephone } \\
\text { (recruitment strategy } \\
\text { B) }\end{array}$ \\
\hline $\begin{array}{l}\text { Presenting } \\
\text { psychological } \\
\text { difficulties and related } \\
\text { needs; expectations } \\
\text { concerning the } \\
\text { ENGAGE intervention; } \\
\text { main distressing } \\
\text { concerns regarding } \\
\text { healthcare utilisation } \\
\text { and productivity losses } \\
\text { related to their child's } \\
\text { cancer }\end{array}$ & $\begin{array}{l}\text { Semistructured } \\
\text { questions }\end{array}$ & & $\checkmark$ & & & & Telephone \\
\hline Depression & PHQ-9 & & $\checkmark$ & $\checkmark$ & $\checkmark$ & $\checkmark$ & $\begin{array}{l}\text { Portal/telephone } \\
\text { Only Portal during } \\
\text { intervention }\end{array}$ \\
\hline Anxiety & GAD-7 & & $\checkmark$ & $\checkmark$ & & $\checkmark$ & Portal/telephone \\
\hline Fear of recurrence & $\begin{array}{l}\text { Structured } \\
\text { question }\end{array}$ & & $\checkmark$ & $\checkmark$ & & $\checkmark$ & Portal/telephone \\
\hline $\begin{array}{l}\text { Fear of serious health } \\
\text { condition }\end{array}$ & $\begin{array}{l}\text { Structured } \\
\text { question }\end{array}$ & & $\checkmark$ & $\checkmark$ & & $\checkmark$ & Portal/telephone \\
\hline $\begin{array}{l}\text { Psychological } \\
\text { inflexibility and } \\
\text { experiential avoidance }\end{array}$ & AAQ-6 & & $\checkmark$ & $\checkmark$ & $\checkmark$ & $\checkmark$ & $\begin{array}{l}\text { Portal/telephone } \\
\text { Only Portal during } \\
\text { intervention }\end{array}$ \\
\hline Depressed inactivity & BADS & & $\checkmark$ & $\checkmark$ & $\checkmark$ & $\checkmark$ & $\begin{array}{l}\text { Portal/telephone } \\
\text { Only Portal during } \\
\text { intervention }\end{array}$ \\
\hline Fatigue & FSS & & $\checkmark$ & $\checkmark$ & & $\checkmark$ & Portal/telephone \\
\hline Quality of life & $E Q-5 D$ & & $\checkmark$ & $\checkmark$ & & $\checkmark$ & Portal/telephone \\
\hline Self-compassion & SCS-SF & & $\checkmark$ & $\checkmark$ & & $\checkmark$ & Portal/telephone \\
\hline Health economics & TiC-P & & $\checkmark$ & $\checkmark$ & & & Portal/telephone \\
\hline
\end{tabular}


Table 3 Continued

\begin{tabular}{|c|c|c|c|c|c|c|c|}
\hline Variable/phenomena & Measure & $\begin{array}{l}\text { Eligibility } \\
\text { interview }\end{array}$ & Baseline & Postassessment & $\begin{array}{l}\text { Weekly } \\
\text { process } \\
\text { evaluation }\end{array}$ & $\begin{array}{l}\text { Six-month } \\
\text { follow-up }\end{array}$ & $\begin{array}{l}\text { Mode of } \\
\text { administration }\end{array}$ \\
\hline $\begin{array}{l}\text { Acceptability of } \\
\text { the intervention; } \\
\text { e-therapist; and } \\
\text { study procedures; } \\
\text { views concerning the } \\
\text { impact of the ENGAGE } \\
\text { intervention. } \\
\text { Non-attendees and } \\
\text { poor attendees are } \\
\text { asked about reasons } \\
\text { for disengaging, } \\
\text { barriers to treatment } \\
\text { and suggestions for } \\
\text { future intervention } \\
\text { development and study } \\
\text { procedures. }\end{array}$ & $\begin{array}{l}\text { Semistructured } \\
\text { questions }\end{array}$ & & & $\checkmark$ & & & Telephone \\
\hline
\end{tabular}

AAQ-6, Acceptance and Action Questionnaire; BADS, Behavioural Activation for Depression Scale; EQ-5D, EuroQol 5-dimension questionnaire; FSS, Fatigue Severity Scale; GAD-7, Generalized Anxiety Disorder 7-item scale; MINI, Mini-International Neuropsychiatric Interview; PCL-5, Post-traumatic Stress Disorder Checklist for DSM-5; PCL-C, Post-traumatic Stress Disorder Checklist-Civilian version; PHQ-9, Patient Health Questionnaire; PTSS, symptoms of post-traumatic stress; SCS-SF, Self-

Compassion Scale-Short Form; TiC-P, Trimbos and Institute of Medical Technology Assessment Cost Questionnaire for Psychiatry.

interventions ${ }^{45-47}$ and qualitative process evaluations. ${ }^{48}$ To explore the non-acceptability of the intervention, study procedures and potential barriers to treatment, non-attendees (parents who do not attend the initial video or telephone assessment session or complete the introductory chapter or any modules) and poor attendees (attend the initial video or telephone assessment with an e-therapist but disengage prior to completion of the introductory chapter and at least five modules alongside the attendance of the 'booster' support session) will be invited to be interviewed. Semistructured interviews with non-attendees and poor attendees will explore reasons for disengaging, barriers to treatment, and examine suggestions for future intervention development and study procedures.

\section{Data analyses}

Data analyses will primarily be descriptive and will address the outcomes relating to the feasibility of the intervention and study procedures. Progression criteria will be used to determine whether revisions should be considered before proceeding to a controlled trial (table 2). ${ }^{22}$

\section{Quantitative analyses}

An adapted CONSORT diagram for pilot and feasibility studies ${ }^{23}$ will be used to illustrate participant flow. Numbers of parents identified via the Swedish Childhood Cancer Foundation and NAVET registry (Swedish Tax Agency) or by advertisements, numbers expressing initial interest, consented, assessed for eligibility, eligible and included, will be reported. The percentages of: (1) parents assessed for eligibility of the total number invited; (2) parents meeting eligibility criteria of the total number invited; and (3) parents enrolled in the study of the total number invited will be calculated with exact 95\% CIs. Reasons for ineligibility, ambiguities regarding eligibility criteria and reasons for non-participation will be reported at each stage.

Follow-up rates and numbers of missing items relating to outcome measures will be calculated with $95 \%$ CIs. In addition, means and SDs for the number of reminders sent via text message, email and telephone will be reported. Potential assessment uncertainties in diagnostic interviews will be reported alongside means and SDs for time taken to complete questionnaires and interviews. Descriptive statistics including the means and SDs or medians and IQRs and change scores for each outcome measurement at the eligibility interview, baseline and post-treatment, and at 6-month follow-up will be reported. Attrition proportions (both intervention and study dropout) will be reported with $95 \%$ CIs.

Means, SDs and frequencies for each Portal activity relating to intervention adherence and use, including log-ins, opened modules and items, completed action plans and use of optional support functions will be reported. Means, SDs and frequencies of parent and e-therapist contact within the Portal, via telephone and/or video will be reported and e-therapist adherence measures will be summarised with means and SDs and collated in total and by e-therapist.

Means and SDs for the length of time taken for parents to work through the intervention and for parents to complete the eligibility interview, baseline, post-treatment (12 weeks) and 6-month follow-up assessments will be reported. In addition, means and SDs will be reported for the length of time e-therapists spend delivering the intervention; for therapist training and 
supervision, and for project personnel to administer the data collection procedures from invitation through follow-up. These data will be used to assess the feasibility of the intervention and study procedures. Potential ambiguities regarding standard safety procedures, types and numbers of measures undertaken to assure patient safety, and types and numbers of unforeseen safety issues will be reported.

\section{Qualitative analyses}

Answers to semistructured interview questions will be recorded, transcribed verbatim, checked for accuracy and analysed using qualitative content analysis. ${ }^{49}$ To increase trustworthiness of the analysis, at least two researchers and parent research partners will be involved in all stages of analysis to increase credibility, and ensure results accurately represent parents' experiences. Disconfirming case analysis ${ }^{50}$ will be conducted to further improve rigour and trustworthiness. Content analysis ${ }^{49}$ will be used to analyse the written communication in the interactive functions of the intervention.

\section{Patient and public involvement statement}

The intervention and study protocol were designed with input from participants using a PAR approach, which was cofacilitated by a member of the research team and a parent of a child successfully treated for cancer. ${ }^{20}$ There was no further involvement in the development of this trial by patients or the public; however, a patient and public involvement panel will be established to work alongside the research team during the course of the study. An end of study report will be developed to communicate study results to all participants. In addition, a study newsletter will be sent to participants via email approximately 6 weeks before post-treatment and 6-month follow-up.

\section{ETHICS AND DISSEMINATION}

The study has been approved by the Regional Ethical Review Board in Uppsala, Sweden (Dnr: 2017/527) and will be conducted in accordance with the Helsinki Declaration, ensuring the welfare and rights of all participants. Confidentiality will be guaranteed and consideration will be given to participants' integrity, dignity and vulnerability. Informed consent will be collected to ensure participants are aware of the conditions of study participation. Participants will be reminded of their rights to withdraw from the study without giving any reason. Participants will be provided with contact information within the study invitation packs for both the principal investigator (coauthor LvE) and the independent Patient Health and Safety Officer for the U-CARE group should they have any cause for concern regarding the conduct of the trial. All data will be handled according to Patient Data Act (2008:355) and the General Data Protection Regulation (EU 2016/679) with all participants assigned a study code to deidentify data and personal information about participants stored separately from deidentified data. Data collected via the Portal will be stored on secure servers at Uppsala University, with personal data and user-generated data stored on separate databases on different servers. The Portal secures deidentification of data and prevents unauthorised persons to connect data from different Portal databases. All other data will be stored in a locked filing cabinet, accessible only to study personnel. Responsible healthcare provider will be U-CARE Healthcare operating according to the Patient Safety Act (2010:659), Patient Data Act (2008:355) and the Health and Medical Services Act (2017:30). Any adverse events or negative effects discovered during the study will be reported following standard U-CARE Healthcare procedures. Assessments are carried out throughout the study to ensure participants in need of more extensive support, than provided within the study, are identified and guided to appropriate healthcare services. Communication within the Portal will be monitored to identify participants at risk of harm to self or signalling a need for more extensive support. Study findings will be published in an open-access journal and via national and international conference presentations.

\section{DISCUSSION}

The ENGAGE intervention was developed in response to previous research demonstrating that a substantial subgroup of parents of children previously treated for cancer report psychological distress in response to their child's disease ${ }^{3-6}$ and/or an unmet need of psychological support. ${ }^{9}$ Psychological distress related to a child's cancer disease causes suffering and costs to the individual parent. ${ }^{51}{ }^{52}$ Findings for other populations show that this kind of distress also is costly for society as a whole due to impacts on healthcare utilisation and productivity loss. ${ }^{53}$ Challenges faced by the Swedish healthcare sector concerning a widening gap between mental health treatment demands and available resources can potentially be addressed using internet-administered interventions supported by cost-effective e-therapists. We have recently published findings demonstrating the clinical effectiveness of an internet-administered psychological, self-help, intervention for parents of children recently diagnosed with cancer. ${ }^{11}{ }^{12}$ However, to the best of our knowledge, there is no published evaluation of the clinical efficacy and cost-effectiveness of such an intervention for psychological distress experienced by parents of children previously treated for cancer.

The study presented in this protocol will examine the feasibility and acceptability of ENGAGE, an internet-administered, guided, CBT-based self-help intervention developed to reduce psychological distress among parents of children previously treated for cancer. Investigating the feasibility and acceptability of complex interventions and study procedures is strongly recommended to estimate important parameters and answer key uncertainties required to inform the design of future definitive controlled trials. ${ }^{21}$ Given the novelty of the intervention, 
and limited number of intervention studies conducted with the target population, assessing the acceptability and feasibility of the intervention and study procedures is of great importance in informing intervention refinements and the planning of a future definitive controlled trial. Should the ENGAGE intervention and procedures be demonstrated to be feasible and acceptable, the intervention will be evaluated in a definitive controlled trial. In turn, should the intervention be demonstrated to be clinically and cost-effective, the aim is to implement the intervention within the standard Swedish healthcare setting.

\section{Study status}

Recruitment is planned to commence during autumn 2018.

Acknowledgements The authors express their gratitude to everyone who has contributed to the development of the intervention and study procedures, especially to the parent and expert research partners and facilitators involved in the development.

Contributors LvE conceived the idea for the project and secured funding. LvE and JW developed the methodology and analysis plan with contributions from MC, $H G$, GL, AR and AW. LvE, MC and AW contributed to the development of the intervention. All authors made substantial contributions to the drafting, critical revision and final approval of the manuscript.

Funding This work was supported by the Swedish Research Council (grant number K2015-99X-20836-08-4), the Swedish Cancer Society (grant number 170709) and the Swedish Childhood Cancer Foundation (grant number PR2017-0005) (main applicant LvE). LvE is the principal investigator. Uppsala University is acting as the study sponsor.

Disclaimer The funding sources had no role in the design of the study and will not have any role during its execution, analyses, interpretation of the data or decision to submit results.

\section{Competing interests None declared.}

Patient consent Not required.

Ethics approval Ethical approval was granted by the Regional Ethical Review Board in Uppsala, Sweden (Dnr: 2017/527).

Provenance and peer review Not commissioned; peer reviewed for ethical and funding approval prior to submission.

Open access This is an open access article distributed in accordance with the Creative Commons Attribution Non Commercial (CC BY-NC 4.0) license, which permits others to distribute, remix, adapt, build upon this work non-commercially, and license their derivative works on different terms, provided the original work is properly cited and the use is non-commercial. See: http://creativecommons.org/ licenses/by-nc/4.0/

(C) Article author(s) (or their employer(s) unless otherwise stated in the text of the article) 2018. All rights reserved. No commercial use is permitted unless otherwise expressly granted.

\section{REFERENCES}

1. Gustafsson G, Kogner P, Heyman M. Childhood cancer incidence and survival in Sweden 1984-2010. Swedish Childhood Cancer Registry. 2013 http://www.forskasverige.se/wp-content/uploads/ ChildhoodCancerlncidenceandSurvivalinSweden1984_2010.pdf (accessed 9 Nov 2017).

2. Pai AL, Greenley RN, Lewandowski A, et al. A meta-analytic review of the influence of pediatric cancer on parent and family functioning $J$ Fam Psychol 2007;21:407-15.

3. Wakefield CE, McLoone JK, Butow P, et al. Parental adjustment to the completion of their child's cancer treatment. Pediatr Blood Cancer 2011:56:524-31.

4. Ljungman L, Cernvall M, Grönqvist $H$, et al. Long-term positive and negative psychological late effects for parents of childhood cancer survivors: a systematic review. PLoS One 2014;9:e103340.
5. Ljungman L, Hovén E, Ljungman G, et al. Does time heal all wounds? A longitudinal study of the development of posttraumatic stress symptoms in parents of survivors of childhood cancer and bereaved parents. Psychooncology 2015;24:1792-8.

6. Bruce M. A systematic and conceptual review of posttraumatic stress in childhood cancer survivors and their parents. Clin Psychol Rev 2006;26:233-56.

7. Wijnberg-Williams BJ, Kamps WA, Klip EC, et al. Psychological adjustment of parents of pediatric cancer patients revisited: five years later. Psychooncology 2006;15:1-8.

8. Steele AC, Mullins LL, Mullins AJ, et al. Psychosocial Interventions and therapeutic support as a standard of care in pediatric oncology. Pediatr Blood Cancer 2015;62:S585-618.

9. Kukkola L, Hovén E, Cernvall M, et al. Perceptions of support among Swedish parents of children after end of successful cancer treatment: a prospective, longitudinal study. Acta Oncol 2017;56:1705-11.

10. Wakefield CE, McLoone J, Butow P, et al. Support after the completion of cancer treatment: perspectives of Australian adolescents and their families. Eur J Cancer Care 2013;22:530-9.

11. Cernvall $M$, Carlbring $P$, Ljungman $L$, et al. Internet-based guided self-help for parents of children on cancer treatment: a randomized controlled trial. Psychooncology 2015;24:1152-8.

12. Cernvall $M$, Carlbring P, Wikman $A$, et al. Twelve-month follow-up of a randomized controlled trial of internet-based guided self-help for parents of children on cancer treatment. $J$ Med Internet Res 2017:19:e273.

13. Stehl ML, Kazak AE, Alderfer MA, et al. Conducting a randomized clinical trial of an psychological intervention for parents/caregivers of children with cancer shortly after diagnosis. $J$ Pediatr Psychol 2009;34:803-16.

14. Knowles SE, Toms G, Sanders C, et al. Qualitative meta-synthesis of user experience of computerised therapy for depression and anxiety. PLoS One 2014:9:e84323.

15. Scott K, Beatty L. Feasibility study of a self-guided cognitive behaviour therapy internet intervention for cancer carers. Aust J Prim Health 2013;19:270-4.

16. Ferwerda $M$, van Beugen $S$, van Burik $A$, et al. What patients think about E-health: patients' perspective on internet-based cognitive behavioral treatment for patients with rheumatoid arthritis and psoriasis. Clin Rheumatol 2013;32:869-73.

17. Craig P, Dieppe P, Macintyre S, et al. Developing and evaluating complex interventions: the new Medical Research Council guidance. Int J Nurs Stud 2013;50:587-92.

18. Ljungman L, Cernvall M, Ghaderi A, et al. An open trial of individualized face-to-face cognitive behavior therapy for psychological distress in parents of children after end of treatment for childhood cancer including a cognitive behavioral conceptualization. PeerJ 2018;6:e4570.

19. Kindon S, Kesby M, Pain R. Participatory Action Research Approaches and Methods: Connecting People, Participation and Place. 1st ed. New York: Routhledge, 2007.

20. Wikman A, Kukkola L, Börjesson H, et al. Development of an internet-administered cognitive behavior therapy program (ENGAGE) for parents of children previously treated for cancer: participatory action research approach. J Med Internet Res 2018;20:e133.

21. Eldridge SM, Lancaster GA, Campbell MJ, et al. Defining feasibility and pilot studies in preparation for randomised controlled trials: development of a conceptual framework. PLoS One 2016;11:e0150205.

22. Thabane L, Ma J, Chu R, et al. A tutorial on pilot studies: the what, why and how. BMC Med Res Methodol 2010;10:1.

23. Eldridge SM, Chan CL, Campbell MJ, et al. CONSORT 2010 statement: extension to randomised pilot and feasibility trials. BMJ 2016;355:i5239.

24. Moore GF, Audrey S, Barker M, et al. Process evaluation of complex interventions: Medical Research Council guidance. BMJ 2015;350:h1258.

25. Chan AW, Tetzlaff JM, Altman DG, et al. SPIRIT 2013 statement: defining standard protocol items for clinical trials. Ann Intern Med 2013;158:200-7.

26. Sheehan DV, Lecrubier $\mathrm{Y}$, Sheehan $\mathrm{KH}$, et al. The Mini-International Neuropsychiatric Interview (M.I.N.I.): the development and validation of a structured diagnostic psychiatric interview for DSM-IV and ICD10. J Clin Psychiatry 1998;59(Suppl 20):22-33.

27. Sim J, Lewis M. The size of a pilot study for a clinical trial should be calculated in relation to considerations of precision and efficiency. $J$ Clin Epidemiol 2012;65:301-8.

28. Bower P, Brueton V, Gamble C, et al. Interventions to improve recruitment and retention in clinical trials: a survey and workshop to assess current practice and future priorities. Trials 2014:15:399. 
29. Barnes M, Wiles N, Morrison J, et al. Exploring patients' reasons for declining contact in a cognitive behavioural therapy randomised controlled trial in primary care. Br J Gen Pract 2012;62:371-7.

30. Farrand $\mathrm{P}$, Woodford J, Llewellyn $\mathrm{D}$, et al. Behavioural activation written self-help to improve mood, wellbeing and quality of life in people with dementia supported by informal carers (PROMOTE): a study protocol for a single-arm feasibility study. Pilot Feasibility Stud 2016;2:42.

31. Ridgway N, Williams C. Cognitive behavioural therapy self-help for depression: an overview. J Ment Health 2011;20:593-603.

32. Farrand P, Woodford J. Impact of support on the effectiveness of written cognitive behavioural self-help: a systematic review and meta-analysis of randomised controlled trials. Clin Psychol Rev 2013;33:182-95.

33. Barazzone N, Cavanagh K, Richards DA. Computerized cognitive behavioural therapy and the therapeutic alliance: a qualitative enquiry. Br J Clin Psychol 2012;51:396-417.

34. Weathers FW, Litz BT, Keane TM, et al. The PTSD Checklist for DSM-5 (PCL-5). National Center for PTSD. 2013 www.ptsd.va.gov (accessed 10 Nov 2017).

35. Weathers FW, Litz BT, Herman DS, et al. The PTSD Checklist (PCL): Reliability, Validity, and Diagnostic Utility. San Antonio (TX), 1993. Paper presented at the 9th Annual Conference of the ISTSS.

36. Kroenke K, Spitzer RL, Williams JB. The PHQ-9: validity of a brief depression severity measure. J Gen Intern Med 2001;16:606-13.

37. Spitzer RL, Kroenke K, Williams JB, et al. A brief measure for assessing generalized anxiety disorder: the GAD-7. Arch Intern Med 2006;166:1092-7.

38. Lundgren T, Parling T. Swedish Acceptance and Action Questionnaire (SAAQ): a psychometric evaluation. Cogn Behav Ther 2017:46:315-26.

39. Raes F, Hoes D, Van Gucht D, et al. The Dutch version of the Behavioral Activation for Depression Scale (BADS): psychometric properties and factor structure. J Behav Ther Exp Psychiatry 2010;41:246-50.

40. Krupp LB, LaRocca NG, Muir-Nash J, et al. The fatigue severity scale. Application to patients with multiple sclerosis and systemic lupus erythematosus. Arch Neurol 1989;46:1121-3.

41. Raes F, Pommier E, Neff KD, et al. Construction and factorial validation of a short form of the Self-Compassion Scale. Clin Psychol Psychother 2011;18:250-5.
42. EuroQol Group. EuroQol--a new facility for the measurement of health-related quality of life. Health Policy 1990;16:199-208.

43. Bouwmans $\mathrm{C}$, De Jong K, Timman R, et al. Feasibility, reliability and validity of a questionnaire on healthcare consumption and productivity loss in patients with a psychiatric disorder (TiC-P). BMC Health Serv Res 2013;13:217.

44. Mitchell N, Hewitt CE, Lenaghan E, et al. Prior notification of trial participants by newsletter increased response rates: a randomized controlled trial. J Clin Epidemiol 2012;65:1348-52.

45. Hind D, O'Cathain A, Cooper CL, et al. The acceptability of computerised cognitive behavioural therapy for the treatment of depression in people with chronic physical disease: a qualitative study of people with multiple sclerosis. Psychol Health 2010;25:699-712.

46. Farrand P, Woodford J, Small F, et al. Behavioural activation self-help to improve depression in people living with dementia: The PROMOTE treatment protocol. NZ J Psychol 2017;46:51-62.

47. Ander M, Wikman A, Ljótsson B, et al. Guided internet-administered self-help to reduce symptoms of anxiety and depression among adolescents and young adults diagnosed with cancer during adolescence (U-CARE: YoungCan): a study protocol for a feasibility trial. BMJ Open 2017;7:e013906.

48. Finning K, Richards DA, Moore L, et al. Cost and outcome of behavioural activation versus cognitive behavioural therapy for depression (COBRA): a qualitative process evaluation. BMJ Open 2017;7:e014161.

49. Graneheim UH, Lundman B. Qualitative content analysis in nursing research: concepts, procedures and measures to achieve trustworthiness. Nurse Educ Today 2004;24:105-12.

50. Patton MQ. Qualitative Evaluation and Research Methods. 2nd edn. Newbury Park CA: Sage, 1990.

51. Mader L, Roser K, Baenziger J, et al. Household income and risk-ofpoverty of parents of long-term childhood cancer survivors. Pediatr Blood Cancer 2017:64:e26456.

52. Lindahl Norberg A, Montgomery SM, Bottai M, et al. Short-term and long-term effects of childhood cancer on income from employment and employment status: a national cohort study in Sweden. Cancer 2017;123:1238-48.

53. Smit F, Cuijpers P, Oostenbrink J, et al. Costs of nine common mental disorders: implications for curative and preventive psychiatry. J Ment Health Policy Econ 2006;9:193-200. 